\title{
Evaluation of Monoclonal Antibody (IgG2bMAb) for Detection of Coproantigen from Experimentally Infected Rats with Strongyloides ratti
}

\author{
Noor Abduhaleem ${ }^{1,2}$, Aliyu Mamuda ${ }^{1,3}$, Tijjani Mustapha ${ }^{1,4^{*}}$, \\ Roslaini Abd Majid ${ }^{1}$, Leslie Than Thian Lung ${ }^{1}$ and Ngah Zasmy Unyah $^{1^{* *}}$ \\ ${ }^{1}$ Department of Medical Microbiology and Parasitology, Faculty of Medicine and Health Sciences, \\ Universiti Putra Malaysia, 43400 UPM, Serdang, Malaysia. \\ ${ }^{2}$ Department of Biology, College of Science, University of Anbar, Anbar, Iraq. \\ ${ }^{3}$ Department of Parasitology and Entomology, Faculty of Veterinary Medicine, Usmanu Danfodiyo \\ University, Sokoto, Nigeria. \\ ${ }^{4}$ Department of Biological Sciences, Faculty of Science, Yobe State University, Damaturu, Nigeria.
}

Authors' contributions

This work was carried out in collaboration among all authors. Author NA designed the study, performed the statistical analysis, wrote the protocol and wrote the first draft of the manuscript. Authors NZU, RAM, AM and LTTL managed the analyses of the study. Author TM managed the literature searches. All authors read and approved the final manuscript.

Article Information

DOI: 10.9734/ARRB/2019/v32i230082

Editor(s):

(1) Dr. J. David Puett, Professor, Department of Biochemistry and Molecular Biology, University of Georgia, Athens, USA.

Reviewers:

(1) J. Y. Peter, University of Abuja, Nigeria. (2) Attahiru Adamu, College of Nursing and Midwifery Gombe, Nigeria. Complete Peer review History: http://www.sdiarticle3.com/review-history/49853

Original Research Article

Received 15 April 2019

Accepted 27 June 2019

Published 03 July 2019

\section{ABSTRACT}

Background and Aim: Highly sensitive and specific diagnostic assay for the detection of Strongyloides is needed due to the intermittent and low concentration of eggs, larvae and adult worms that can be found in a faecal specimen. In some cases, repeated sampling of the faecal specimen is required to obtain satisfactory and reliable results. The aim of the study is to develop and evaluates monoclonal antibody-based Sandwich ELISA for the detection of coproantigen associated with Strongyloides infection using $S$. ratti as a model.

Place and Duration of Study: Department of Medical Microbiology and Parasitology, Faculty of Medicine and Health Sciences, University Malaysia, Between September 2018 and 
March 2019.

Methodology: The monoclonal antibody was raised against a soluble antigen of the infective filariform larvae (iL3) of S. ratti. The monoclonal antibody produced (IgG2bMAb) was evaluated for cross-reactivity against homologous and heterologous helminth antigens such as excretorysecretory (ES), infective larvae (iL3) and coproantigen of $S$. ratti, adult worms of $A$. caninum, $A$. suum, T. canis and T. cati.

Results: An IgG2bMAb was observed to react with $30 \mathrm{kDa}$ proteins associated with all homologous antigen from iL3, ES and coproantigen of $S$. ratti and cross-reacted with one heterologous antigen from adult worm of $A$. caninum at the same molecular weight. There was no cross-reaction observed with other heterologous antigens from adult worms of $T$. canis, $T$. cati and $A$. suum. The sensitivity of IgG2bMAb for the detection of $S$. ratti was $85 \%$ in Sandwich ELISA. Crossreaction was observed with hookworm antigen that caused by $A$. caninum in Western immunoblotting.

Conclusion: The results indicated that IgG2b have an immunodiagnostic property as IgG2bMAb and was able to detect antigens from coproantigen related to $S$. ratti with $85 \%$ sensitivity based on Sandwich ELISA) even though cross-reaction was observed with $A$. caninum. These findings will be very useful to tackle many cases of multiple worms' infections such as both strongyloidiasis and hookworm. Therefore, we recommend that further evaluation and study in the human area where multiple infections can be common should be carried out.

Keywords: Monoclonal antibody; Strongyloides ratti.

\section{INTRODUCTION}

Strongyloidiasis is a parasitic intestinal infection caused by $S$. stercoralis and $S$. fuelleborni in humans with $S$. stercoralis being the most common parasite associated with human infections [1]. This parasite is responsible for some of the zoonotic infections worldwide and is predominantly found in tropical and subtropical countries such as in Africa and Southeast Asia, where the disease is endemic [2]. Despite the severity of the infection among immunosuppressed and elderly patients, the disease is still considered as one of the most neglected tropical diseases [3]. Fatal hyperinfection associated with disseminated infection has been widely documented, especially in the immunosuppressed patients undergoing therapy with a corticosteroid $[4,5,6]$. Strongyloidiasis is still one of the most difficult parasitic infections to diagnose due to intermittent and low parasite concentration apart from being misdiagnosed as hookworm infection [7]. The commonly used diagnostic methods are based on clinical manifestations or demonstration of the parasite using standard parasitological techniques such as agar plate, Baermann and microscopy [8]. Antigen-based serological and molecular-based methods have been used for detection of strongyloidiasis $[9,10,11]$. In this study, the use of monoclonal antibody-based Sandwich ELISA for the detection of coproantigen of $S$. ratti was evaluated.

\section{MATERIALS AND METHODS}

\subsection{Preparation of Soluble Antigens from Homologous and Heterologous Helminthes}

ES and iL3 of S. ratti were harvested from the in vitro culture of iL3 in Dulbecco's Modified Eagle's Medium (DMEM)-serum free medium (Merck, USA) as described earlier [12]. The adult worms of $A$. suum, T. cati, $T$. canis and $A$. caninum were collected from the intestines of an infected pig, kittens, puppies and dogs respectively. Soluble antigens were prepared from the adult worms by freezing and thawing in liquid nitrogen. Then, the as-prepared antigens were homogenised and sonicated in cold phosphate buffered saline solution with $\mathrm{pH} 7.2$ [13]. The soluble antigens were then separated by centrifugation at 5000 $\mathrm{rpm}$ at $40^{\circ} \mathrm{C}$ and protein concentrations were determined using Bradford Protein Assay Kit (Thermo Fisher Scientific, USA) based on the manufacturer instructions. The antigens were then supplemented with protease inhibitor (Merck, USA) and stored in aliquots at $-80^{\circ} \mathrm{C}$.

\subsection{Immunization of Mice}

The monoclonal antibody was developed against the soluble antigen of iL3 larvae of $S$. ratti [14]. Blood samples were collected every two weeks from the tail vein of each mouse before and after immunisation and were used as controls for positive and negative sera $[15,16]$. Five to eight 
weeks old female Balb/c mice were immunised intraperitoneal with $50 \mu \mathrm{g} / \mathrm{mL}$ of the soluble antigen emulsified in Complete Freund's adjuvant (Merck, USA). At day 14 and 21, boosters were given with the same antigen concentration $(50 \mu \mathrm{g} / \mathrm{mL})$ emulsified in an equal volume of Incomplete Freund's adjuvant (Merck, USA). Blood samples were collected at 21 days after booster immunisation and were analysed for antibody titers against similar antigen using Indirect ELISA $[17,18,19]$. The mouse with the highest antibody titer was selected for the development of hybridoma cells. Parental myeloma cells of Balb/c mice origin, Sp2/0-Ag14 (ATCC $₫$ CRL-1581 ${ }^{\mathrm{TM}}$ ) was used to stabilize hybridoma cells for the continuous secretion of monoclonal antibodies [15,16]. The cells were cultured in $5 \mathrm{~mL}$ of DMEM (Merck, USA), containing $10 \%$ fetal bovine serum (Meck, USA) in a $25 \mathrm{~cm} 3$ tissue culture flask (Thermoscientific, USA). Then, the cells were incubated in a $5 \%$ $\mathrm{CO} 2$ at $37^{\circ} \mathrm{C}$ (Galaxy B+ Incubator, RS Biotek, Scotland). Similarly, the spleen of the selected mice was aseptically removed and washed in 35 x $10 \mathrm{~mm}$ sterile tissue culture dishes (Thermoscientific, USA), containing a DMEMserum-free medium. The spleen was disaggregated into a single cell suspension by flushing with a three $\mathrm{mL}$ sterile syringe and a 25 $G$ needle containing a DMEM-serum-free medium. In preparation for the cell fusion, the $2 \mathrm{x}$ 107 of the parental myeloma cell and $6 \times 107$ viable splenocytes were mixed in a ratio of $1: 3$ $[14,16]$.

\subsection{Fusion of Hybridoma Cells}

Fusion was done in a $50 \mathrm{~mL}$ conical tube and was centrifuged for 10 minutes at $1500 \mathrm{rpm}$. The resulting supernatant was completely discarded [11]. Fusion was performed by disrupting the cell pellets by gently tapping the bottom of the tube. 1 $\mathrm{mL}$ of $50 \%$ polyethylene glycol (Merck, USA) was continuously added to the tube over the period of 1 minute under stirring. Then, $4 \mathrm{~mL}$ of DMEMserum-free medium was added to the fusion mixture with continuous gentle stirring over a period of 4 minutes and the tube was incubated for 15 minutes in water bath at $37^{\circ} \mathrm{C}$. About 30 $\mathrm{mL}$ of Complete Growth Medium (STEMCELL Technologies, USA) was slowly added to the tube and the cells were centrifuged at $1500 \mathrm{rpm}$ for 10 minutes. The fused cells were resuspended in $10 \mathrm{~mL}$ of Hybridoma Recovery Medium (STEMCELL Technologies, USA) and transferred to a $75 \mathrm{~cm}^{2}$ tissue culture flask containing new $20 \mathrm{~mL}$ of Hybridoma Recovery
Medium (STEMCELL Technologies, USA) and incubated for 3 days in a $5 \% \mathrm{CO} 2$ at $37^{\circ} \mathrm{C}$ (Galaxy B+ Incubator, RS Biotek, Scotland). The hybrid clones were then selected in a culture medium supplemented with hypoxanthineaminopterin-thymidine (HAT) and sub-cloned by limiting dilutions [16]. Indirect ELISA was then used to screen for antibodies producing hybrid cells for continuous in vitro culture $[19,18,20]$.

\subsection{Isotyping of Monoclonal Antibody}

Indirect ELISA was used to confirm the antibodysecreting hybridoma cells $[19,18,20]$ and Western immunoblot [21,22]. Intraperitoneal injection of $1 \times 106$ hybridoma cells was done for the production of ascites fluid. The ascites fluid was collected and centrifuged at $5000 \mathrm{rpm}$ at $40^{\circ} \mathrm{C}$ for 15 minutes and the supernatant was collected and stored at $-20^{\circ} \mathrm{C}$. Isotyping of the supernatant from the in vitro culture of hybridoma cells and ascites fluid were done using Mouse Monoclonal Antibody Isotyping Kit (Merck, USA) based on the manufacturer instructions.

\subsection{Mycoplasma Test}

Supernatant from the in vitro culture of hybridoma cells and ascites fluid was tested for contamination with mycoplasma using Universal Mycoplasma Detection Kit (ATCC, USA) based on the manufacturer instructions.

\subsection{Indirect ELISA with Homologous and Heterologous Antigens}

The reaction of the monoclonal antibody against homologous antigens (iL3, ES and coproantigen of $S$. ratti) and heterologous antigens (from the adult worms of $A$. suum, $T$. cati, $T$. canis and $A$. caninum) were done using Indirect ELISA $[19,18,20]$. $0.5 \mu \mathrm{g} / \mathrm{mL}$ of each heterologous antigen was coated on the ELISA plates separately (Thermo Fisher, USA) and was incubated at $40^{\circ} \mathrm{C}$ overnight. $100 \mu \mathrm{L}$ of Blocking Reagent (Merck, USA) were added to the coated ELISA plates and were incubated for 30 minutes at $37^{\circ} \mathrm{C}$. The coated ELISA plates were washed three times with $100 \mu \mathrm{L}$ PBS-Tween $20(\mathrm{pH}$ of 7.2) [19]. Then, the plates were reacted with monoclonal antibody and incubated for 30 minutes at $37^{\circ} \mathrm{C}$. The plates were then washed three times with $200 \mu \mathrm{L}$ PBS-Tween $20(\mathrm{pH}$ 7.2) and $100 \mu \mathrm{L}$ ALP Goat-anti mouse IgG (Merck, USA) was then added as secondary antibody and incubated at $37^{\circ} \mathrm{C}$ for 45 minutes. PNitrophenyl-phosphate (pNPP) (Thermo Fisher, USA) was used as the substrate at $405 \mathrm{~nm}$ 
(Dynex Technologies, USA) after 30 minutes incubation at room temperature. The reaction was stopped after adding $100 \mu \mathrm{L}$ of $0.75 \mathrm{M}$ $\mathrm{NaOH}$.

\subsection{Western Immunoblotting with Homologous and Heterologous Antigens}

Each homologous and heterologous antigen was loaded in the separated wells of the SDS-PAGE system [23]. and then the gel was run for 1-2 $h$ at $100 \mathrm{~V}$ (Mini Trans-Blot, Bio-Rad USA). The antigens were then transferred to an Immobilon ${ }^{\circledR}$ PVDF Membranes (Merck, USA) using a Semidry blotter (Merck, USA) as instructed by the manufacturer. The membranes were then soaked in a blocking solution, incubated with agitation for 1 hour and washed three times with agitation in PBS for 10 minutes. After that, the resulting membranes were then reacted with the monoclonal antibody and incubated with agitation for one hour at room temperature and washed three times with agitation in PBS for 10 minutes. $[22,21]$. Next, the membranes were further incubated in ALP Goat-anti mouse IgG (Merck, USA) as a secondary antibody with agitation for 3 hours at room temperature, washed three times with agitation in PBS for 10 minutes and incubated with agitation in SIGMAFAST ${ }^{\mathrm{TM}}$ $B C I P \circledast / N B T$ (SIGMA-ALDRICH, USA) for another 30 minutes at room temperature. The reaction was terminated by washing the membranes with distilled water.

\subsection{Preparation of Rat Fecal Specimens for Sandwich ELISA}

Fifty [50] faecal samples collected from 20 experimental-based inoculated rat with $S$. ratti [12]. as well as 30 clean and uninfected rats as, normal controls were used for the sensitivity test and standardisation of Sandwich ELISA. Faecal specimens collected from each rat were mixed with $1 \mathrm{~mL}$ PBS $(\mathrm{pH} 7.2)$ in an Eppendorf tube. The mixture was then homogenised and spin at $5000 \mathrm{rpm}$ for 10 minutes. The resulting supernatant was collected into new Eppendorf tubes and the pellets were discarded [26]. The supernatant was kept at $-80^{\circ} \mathrm{C}$ before further analysis was conducted using Sandwich ELISA $[24,25]$.

\subsection{Sandwich ELISA for Detection Coproantigen of S. ratti}

ELISA plates were coated with $5 \mu \mathrm{g} / \mathrm{mL}$ of rabbit polyclonal antibodies that raised against iL3 larvae of $S$. ratti and incubated overnight at $40^{\circ} \mathrm{C}$. $100 \mu \mathrm{L}$ of Blocking Reagent (Merck, USA) was added and then were incubated for 30 minutes at $37^{\circ} \mathrm{C}$. The plates were then washed three times with $200 \mu \mathrm{L}$ PBS-Tween $20(\mathrm{pH}$ 7.2). Then, $100 \mu \mathrm{L}$ of supernatant from faecal specimen was added and incubated for 1 hour at $37^{\circ} \mathrm{C}$. The plates were then washed three times with $200 \mu \mathrm{L}$ PBS-Tween $20 \quad(\mathrm{pH}$ 7.2), reacted with monoclonal antibody, incubated for 30 minutes at $37^{\circ} \mathrm{C}$ [25] and washed three times with $200 \mu \mathrm{L}$ PBS-Tween $20(\mathrm{pH} 7.2)$ before added $100 \mu \mathrm{L}$ ALP Goat-anti mouse IgG (Merck, USA) that acts as secondary antibody. The mixture was then incubated at $37^{\circ} \mathrm{C}$ for 45 minutes. P-Nitrophenylphosphate (pNPP) (Thermo Fisher, USA) was added after incubated for 30 minutes at room temperature. The reaction was terminated by adding $100 \mu \mathrm{L}$ of $0.75 \mathrm{M} \mathrm{NaOH}$ into the mixture [24].

\section{RESULTS}

In this study, three monoclonal antibodies denoted as S1, S2 and S3 were developed against the soluble antigen of iL3 larvae of $S$. ratti (Fig.1). Monoclonal antibody S3 was selected for this study and renamed as IgG2bMAb (monoclonal S1 and S2 were not used in this study and were cryopreserved). Characterization of the immunoglobulin subclasses based on the Monoclonal Antibody Isotyping Kit (Merck, USA) showed that monoclonal antibody S1, S2 and S3 were from the immunoglobulin sub-classes of IgG1, IgG2a and IgG2b respectively (Fig. 1 ).

Western Immunoblotting analysis of IgG2bMAb against the two homologous antigens of ES and iL3 of $S$. ratti confirmed that IgG2bMAb was able to react with both the homologous antigens. IgG2bMAb reacted strongly to the antigen from iL3 compared to ES from the same larvae of $S$. ratti (Fig. 2). Western immunoblotting analysis for the cross-reactivity with other species of heterologous intestinal helminth was done. Heterologous antigens such as the adult worm soluble antigens from $T$. canis (Tcn), T. cati (Tct), $A$. caninum (Ac) and $A$. suum (As) were tested against IgG2bMAb. IgG2bMAb was observed to cross-reacted strongly at $30 \mathrm{kDa}$ with $A$. caninum (Ac) antigen by producing similar band intensity with the positive control antigen from iL3 of $S$. ratti (Sr) used in this study (Fig. 3).

There was no visible cross-reaction detected with $T$. canis (Tcn), T. cati (Tct) and A. suum (As) 
antigens by IgG2bMAb (Fig. 3). IgG2bMAb was further tested in Sandwich ELISA for the immunodiagnostic detection of coproantigen of $S$. ratti in rat's faecal specimen. Thirty clean and uninfected rats were used as the negative control for the standardisation of the cut-off-point used in the Sandwich ELISA (Mean \pm 3 SD $=0.266$ ) (Fig.4). Twenty positively inoculated rat's faecal specimens (previously subcutaneously inoculated with $1 \times 105$ iL3 larvae) [12]. was used for the sensitivity study in Sandwich ELISA. In this study, IgG2bMAb detected 17 positive coproantigen from 20 inoculated faecal specimens of experimentally inoculated rats with iL3 of $S$. ratti with three false negatives detected below 0.266 (Fig. 4). Thus, the sensitivity of IgG2bMAb based Sandwich ELISA for detection of coproantigen of $S$. ratti in rat's faecal specimens was $85 \%$ as calculated from the detection rate of $17 / 20 \times 100$ (Fig.4).
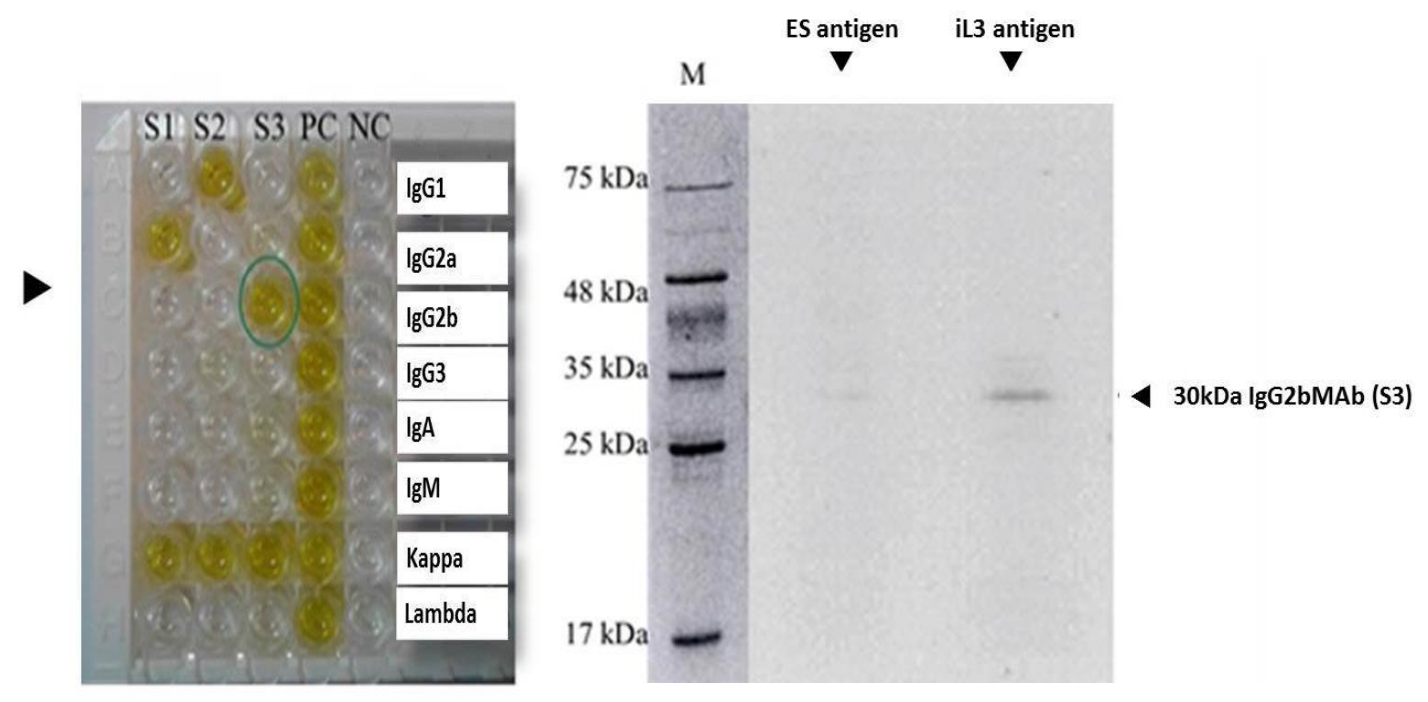

Fig. 1. Characterization of the immunoglobulin sub-classes based on isotyping kit
Fig. 2. Western immunoblotting analysis of IgG2bMAb against ES and iL3 of S. ratti

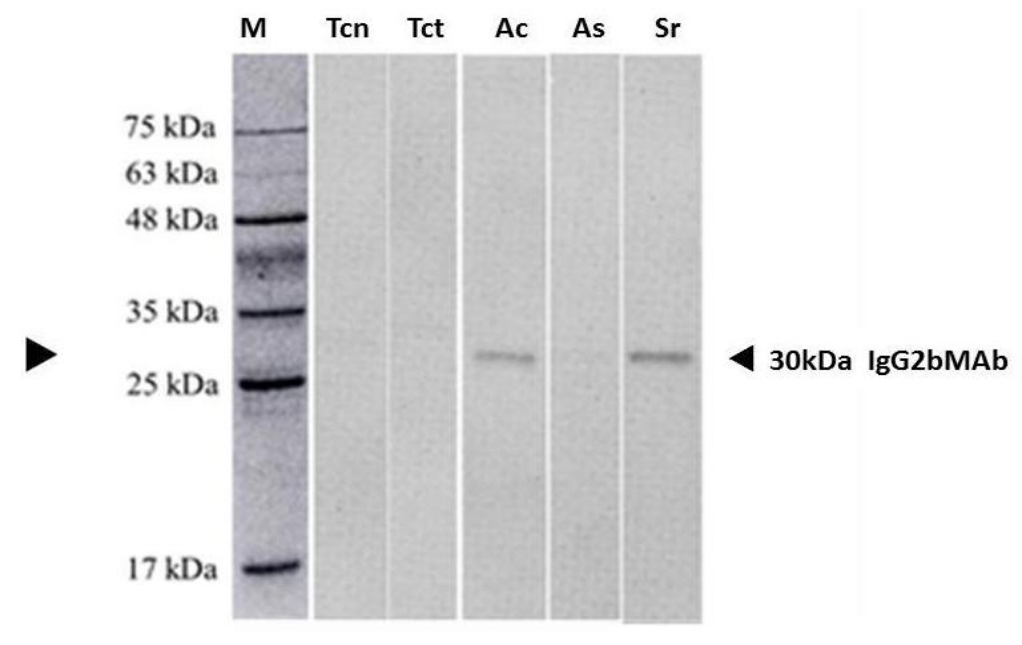

Fig. 3. Western immunoblotting analysis for the cross-reactivity with other species of heterologous intestinal helminth 


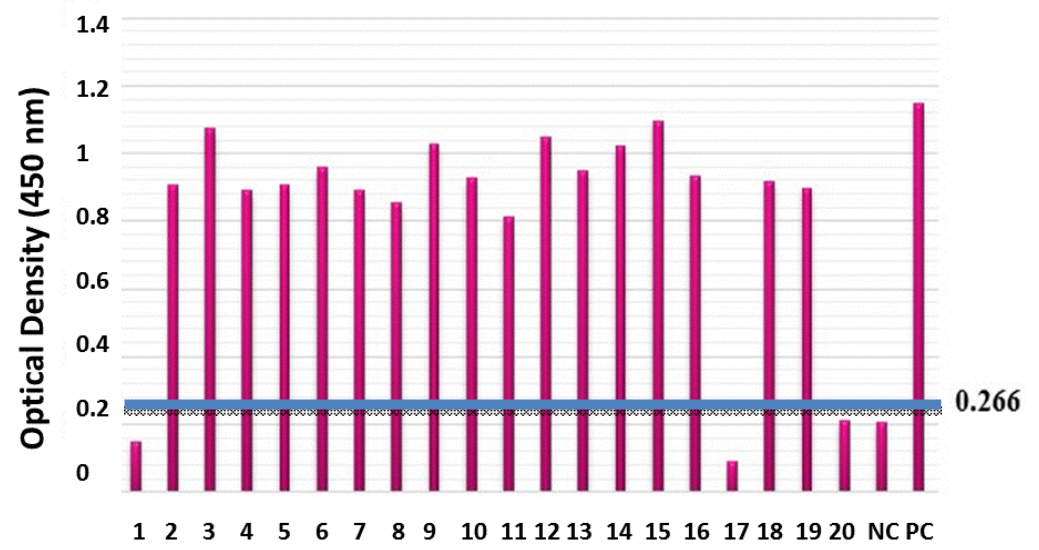

Sample Number

Fig. 4. Sandwich ELISA for the immunodiagnostic detection of coproantigen of $S$. ratti in rat's faecal specimen

$N=20$ infected rat wit $S$. ratti sandwich ELISA, positive detected $=17$ sandwich ELISA. False negative detected $=3$, Sensitivity of IgG2bMAb

\section{DISCUSSION}

Strongyloidiasis is frequently underdiagnosed because many of the cases are asymptomatic and current diagnostic methods are lacking sensitivity. The early detection of this disease is still one of the major obstacles in the infection prevention. Conventional diagnostic methods are insensitive due to the poor and inconsistent parasite load in stool samples. World Health Organization estimated that about 30-100 million people are infected worldwide, and the precise data is still remained unknown, especially in the endemic countries which are associated with poor sanitary conditions. Acute and chronic infections have been reported widely in children, elderly and the immunosuppressed patients. Fatal infections mostly occur in the immunosuppressed patient associated with corticosteroids chemotherapy. In this group of patients, the majority of the cases are underdiagnosed due to asymptomatic infection and lack of defined symptoms, hence resulting in fatal disseminated infection. In this study, $S$. ratti was used to represent $S$. stercoralis based on the morphological and similarity in genome up to $80 \%$. Three monoclonal antibodies (S1, S2 and S3) were produced and characterizations of the antibody isotypes were $\lg G 1, \lg G 2 a$ and $\lg G 2 b$ for S1, S2 and S3 respectively (Fig. 1). S3 (later renamed as monoclonal IgG2bMAb) was chosen to be evaluated in the monoclonal IgG2bMAbbased Sandwich ELISA for detection of coproantigen from $S$. ratti in faecal specimens based on experimental inoculated Wistar rats ( $\mathrm{S} 1$ and S2 were cryopreserved). To our knowledge, this is the first study utilises IgG2b in the immunological detection of helminth's coproantigen in the faecal specimen.

Western immunoblotting confirmation for the detection of monoclonal IgG2bMAb against the prepared ES and iL3 antigens of $S$. ratti was conducted. The result showed that monoclonal IgG2bMAb was able to bind with $30 \mathrm{kDa}$ protein of iL3 soluble antigen but low binding with ES antigen of $S$. ratti (Fig. 2). The weak binding with ES protein was probably associated with the fluctuation of secreted ES by iL3 and the establishment of patency for serological detection of ES protein that was previously estimated around 6 to 7 weeks [27]. This resulted in a low concentration of ES protein and detection with monoclonal IgG2bMAb. Nevertheless, this experiment confirmed that IgG2bMAb was able to detect the presence of $S$. ratti immunogenic protein at $30 \mathrm{kDa}$ (Fig. 2). Another study also confirmed that serum IgG reactivity against iL3 of $S$. stercoralis immunogenic proteins was at $28 \mathrm{kDa}, 31 \mathrm{kDa}$ and $41 \mathrm{kDa}$ which were associated with a high quantity of $S$. stercoralis worm [28]. In our study, the $30 \mathrm{kDa}$ detected by monoclonal IgG2bMAb was between $28 \mathrm{kDa}$ and $31 \mathrm{kDa}$, which was in agreement with the study.

Multiple intestinal helminths infection has been reported in endemic areas. These resulted in 
cross-reaction due to similar binding epitopes and may produce false positive results in an immunodiagnostic test [29,30,31]. Hookworm infection caused by Ancylostoma spp and Necator spp. was known to cross-reacted with Strongyloidiasis in an immunodiagnostic test $[32,33,34]$. To observe if similar cross-reaction occurs with monoclonal IgG2bMAb, heterologous antigens from commonly known intestinal helminths such as $T$. canis (Tcn), T. cati (Tct), $A$. caninum $(A c)$ and $A$. suum $(A s)$ were prepared. This is to evaluate if monoclonal IgG2bMAb cross-reacted with these intestinal helminths apart from $S$. ratti that may produce false positive results. In Western immunoblotting, monoclonal IgG2bMAb only cross-reacted with the antigen from the adult worm of $A$. caninum (Ac) at 30 $\mathrm{kDa}$ (Fig. 3). Multiple intestinal helminth is commonly affected children, especially in rural areas in the developing world due to poor hygiene $[35,36,37]$. The cross-reactivity with $A$. caninum suggests that monoclonal IgG2bMAbbased Sandwich ELISA can be used to detect multiple helminth infections, especially from the patient faecal specimen with multiple intestinal helminth infections such as both Strongyloides spp. and Ancylostoma spp. (Fig. 3).

The sensitivity of monoclonal IgG2bMAb-based Sandwich ELISA for detecting coproantigen of $S$. ratti was evaluated with twenty faecal specimens that were positively confirmed being infected rats with S. ratti (Fig.4). The cut-off-point was determined based on Optical Density (OD) reading at 0.266 of Mean $\pm 3 S D$ of thirty uninfected normal control rats with monoclonal IgG2bMAb-based Sandwich ELISA. OD reading above 0.266 was considered positive for the presence of coproantigen of $S$. ratti, whereas OD reading below 0.266 was taken as negative. Monoclonal IgG2bMAb-based Sandwich ELISA was able to detect seventeen positives out of the twenty known infected rats used for this sensitivity test (Fig. 4). Thus, the sensitivity of IgG2bMAb-based Sandwich ELISA for detection of coproantigen of $S$. ratti in rat's faecal specimens was $85 \%$. Three faecal specimens from this group with OD reading below 0.266 were taken as false negative as these specimens were from known positive infected rats. These low OD reading most probably due to low parasite load in a faecal specimen of these three rats. The application of the soluble antigens of iL3 larvae of $S$. ratti used in this study for the production of monoclonal IgG2bMAb was able to detect $30 \mathrm{kDa}$ coproantigens from all the three developmental stages of $S$. ratti which are the eggs, larvae and adults' stages in a faecal specimen. This is a significant finding as not all the stages of $S$. stercoralis can be seen in a faecal specimen that was sent to the diagnostic laboratory [32,33].

\section{CONCLUSION}

In this study, IgG2b proved to have an immunodiagnostic property as IgG2bMAb was able to detect antigens from coproantigen related to $S$. ratti (sensitivity was $85 \%$ based on Sandwich ELISA) even though cross-reaction was observed with $A$. caninum. These findings will be very useful to tackle many cases of multiple worms' infections such as both strongyloidiasis and hookworm but need further evaluation and study in the endemic area where multiple infections can be common.

\section{ETHICAL APPROVAL}

The used of animal in this study was approved by Institutional Animal Care and Use Committee, Universiti Putra Malaysia (UPM/IACUC/AUPR026/2015).

\section{COMPETING INTERESTS}

Authors have declared that no competing interests exist.

\section{REFERENCES}

1. Meruyert B, Harriet W, Kirstin R. Strongyloidiasis: A Disease of Socioeconomic Disadvantage. International Journal of Environmental Research \& Public Health. 2016;512(13): 1-15.

2. Genta RM. Global prevalence of strongyloidiasis: Critical review with epidemiologic insights into the prevention of disseminated disease. Reviews of Infectious Diseases. 1998;11(5):755-67.

3. Venkataramana K, Adnan BB. Human Strongyloidiasis: An insight in to a Neglected Tropical Parasitic Disease. Translational Biomedicine. 2015;6(4):1-7.

4. Hassan T, Muhammad UK, Pavithra R, Bharat B, Masooma N, Ajsza M, Cosmina Z, Ariyo I, Anil D, Sridhar C. Anaemia, intractable vomiting, chronic diarrhoea and syndrome of inappropriate antidiuretic secretion: A diagnostic dilemmadisseminated strongyloidiasis in a patient with newly diagnosed HTLV infection-case 
report and review of the literature. Medicine. 2017;96(52):1-7.

5. Richelle GW, Ernesto AA, Wencheng L, Aimee W, Elizabeth P. Case report: Strongyloides stercoralis hyperinfection in a patient with chronic lymphocytic leukemia. The American Society of Tropical Medicine and Hygiene. 2017;97(5):1629-1631

6. Dina AZ, Lichao ZA, Michelle E, Scott AU, Wei-Han W. Analysis of monoclonal antibody sequence and post-translational modifications by time-controlled proteolysis and tandem mass spectrometry. Molecular and Cellular Proteomics. 2016;15(4):14791488

7. Afzal A, Siddiqui and Steven LB. Diagnosis of Strongyloides stercoralis Infection. Clinical Infectious Diseases. 2001;33(7):1040-7.

8. Francisco HC, Leticia A. A simple modification of the baermann method for diagnosis of Strongyloidiasis. Memórias do Instituto Oswaldo Cruz. 2001;96(6):805807.

9. Fernando S, Elena S, Adria'n SM, Jose' MS, Esperanza $R$, Albert $P$, Israel $M$. Usefulness of strongyloides stercoralis serology in the management of patients with eosinophilia. American Journal of Tropical Medicine \& Hygiene. 2014;90(5): 830-834.

10. Luciana PS, Ivanildes SCB, Andréia BPL, Foued SE, Dulcinéa MBC, Julia MCC. Memórias do Instituto Oswaldo Cruz. 2003;98(5):687-691.

11. Jaco J, Verweija $M$, Canalesb KP, Juventus Z, Eric AT, Brienena AM, Poldermana, Lisette VL. Molecular diagnosis of Strongyloides stercoralis in faecal samples using real-time PCR. Transactions of the Royal Society of Tropical Medicine and Hygiene. 2010;103: 342-6.

12. Mahmuda A, Al-Zihiry KJK, Roslaini AM, Rukman $\mathrm{AH}$, Abdulhaleem $\mathrm{N}$, Bande $\mathrm{F}$. Histopathological confirmation of disseminated larvae (iL3) of Strongyloides ratti in an immunosuppressed Wistar rat. Tropical Biomedicine. 2017;34(1):212-223.

13. Rafaella FQG, Watson HMV, Silva M, Suedali VBB, Elizandra GR, Edward O, Paulo MZC. Antigens of worms and eggs showed a differentiated detection of specific $\lg G$ according to the time of Schistosoma mansoni infection in mice.
Revista da Sociedade Brasileira de Medicina Tropical. 2012;45(4):505-509.

14. Köhler G, Milstein C. Continuous cultures of fused cells secreting antibody of predefined specificity. Nature. 1975;256: 495-497.

15. Hanack K, Messerschmidt K, Listek M. Antibodies and selection of monoclonal antibodies. Böldicke T. (eds) Protein Targeting Compounds. Advances in Experimental Medicine and Biology. 2016; 917:11-22.

DOI: 10.1007/978-3-319-32805-8_2.

16. Wayne M, Yokoyama, Michelle C, Gary DS, Diane M. Production of monoclonal antibodies. Current Protocol. 2006;74(1): 2.5.1-2.5.25.

17. Lin AV. Indirect ELISA. Methods in Molecular Biology. 2015; 1318:51-9.

18. Lequin, R. M. Enzyme Immunoassay (EIA)/Enzyme-Linked Immunosorbent Assay (ELISA). Clinical Chemistry. 2015; 51(12):2415-8.

19. Engvall E, Perlmann P. (Enzyme-linked Immunosorbent Assay (ELISA) Quantitative Assay of Immunoglobulin G. Immunochemistry. 1971;8(9): 871-4.

20. Lin AV. Indirect ELISA. Methods in Molecular Biology. 2015;1318:51-9.

21. Mahmood T, Yang PC. Western Blot: Technique, Theory and Trouble Shooting. North American Journal of Medical Sciences. 2012;4(9):429-434.

22. Towbin $\mathrm{H}$, Staehelin $\mathrm{T}$, Gordon J. Electrophoretic transfer of proteins from polyacrylamide gels to nitrocellulose sheets: procedure and some applications. Proceedings of the National Academy of Sciences USA. 1979;76(9):4350-54.

23. Sambrook J, Fritsch EF, Maniatis T. Molecular cloning: A laboratory manual. New York: Cold spring harbor laboratory pres; 1989.

24. Kragstrup TW, Vorup JT, Deleuran B, Hvid M. A simple set of validation steps identifies and removes false results in a sandwich enzyme-linked immunosorbent assay caused by anti-animal IgG antibodies in plasma from arthritis patients. Springer Plus 2. 2013;(1):263.

DOI:10.1186/2193-1801-2-263.

25. Schmidt SD, Mazzella MJ, Nixon RA, Mathews PM. A $\beta$ measurement by enzyme-linked immunosorbent assay. Methods in Molecular Biology. 2012;849: 507-27. 
26. Nageswaran C, Craig PS, Devaney E. Coproantigen detection in rats experimentally infected with Strongyloides ratti. Parasitology. 1994;108(3):335-42.

27. Uparanukraw P, Phonogsri S, Morakote N. Fluctuations of larval excretion in Strongyloides stercoralis infection. American Journal of Tropical Medicine \& Hygiene. 1999;60:967-73.

28. Conway DJ, Atkins NS, Lillywhite JE, Bailey JW, Robinson RD, Lindo JF. Immunodiagnosis of Strongyloides stercoralis infection: A method for increasing the specificity of the indirect ELISA. Transaction of the Royal Society of Tropical Medicine and Hygiene. 1993;87: 173-176.

29. Viscidi $R$, Laughon $B E$, Hanvanich $M$, Bartlett JG, Yolken RH. Improved enzyme immunoassays for the detection of antigens in faecal specimens: Investigation and correction of interfering factors. Journal of Immunological Methods. 1984; 67:129-143.

30. Hanvanich $M$, Viscidi $R$, Laughon BE, Bartlett JG, Yolken RH. Stool desorbing activity: A possible cause of false-positive reactions in competitive enzyme immunoassays. Journal of Clinical Microbiology. 1985;21:184-188.

31. Pablo PY, Margaret K, Robert HG, Juliana C, Caryn B, Cesar BC, Maribel PO, Carmen M, Graciela MS, Bevelle W, James WFL, Carlos VO. Seroepidemiology of strongyloidiasis in the Peruvian Amazon. American Journal of Tropical Medicine \& Hygiene. 2016;74(1):97-102.

32. Alex M, Sykes, James S, McCarthy. A coproantigen diagnostic test for strongyloides infection. PLOS Neglected Tropical Diseases. 2011;5(2):e955;1-8.

33. Ana RM, Peter C, Zeno B, Dora B, Eduardo G, Jose Mun. The laboratory diagnosis and follow up of Strongyloidiasis: A systematic review. PLOS Neglected Tropical Diseases. 2013;7(1):1-10. e2002.

34. Armelle F, Virak K, Fabian S, Penelope V, Frédérique $C$, Hanspeter $M$, Sinuon $M$, Peter O. Strongyloides stercoralis and hookworm co-infection: Spatial distribution and determinants in Preah Vihear Province, Cambodia. Parasites \& Vectors; 2018.

35. Brooker S, Miguel EAS, Moulin Al, Luoba Bundy AP, Kremer M. Epidemiology of single and multiple species of helminth infections among school children in Busia District, Kenya. East African Medical Journal. 2000;77(3):157-161.

36. Drake LJ, Bundy DAP. Multiple helminth infections in children: Impact and control. Parasitology. 2001;122:73-81. DOI: 10.1017\} S0031182000017662.

37. Jeffrey B, Simon B, Marco A, Stefan MG, Alex L, David D, Peter JH. Soil-transmitted helminth infections: Ascariasis, trichuriasis, and hookworm. Lancet. 2006;367:1521-32.

(c) 2019 Abduhaleem et al.; This is an Open Access article distributed under the terms of the Creative Commons Attribution License (http://creativecommons.org/licenses/by/4.0), which permits unrestricted use, distribution, and reproduction in any medium, provided the original work is properly cited. 